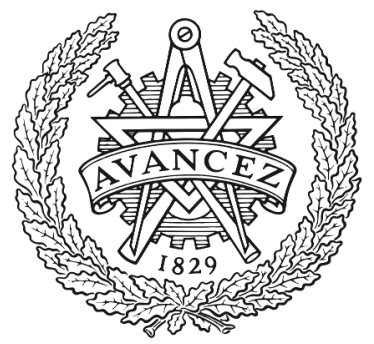

CHALMERS

UNIVERSITY OF TECHNOLOGY

\title{
Plasma solenoid driven by a laser beam carrying an orbital angular momentum
}

Downloaded from: https://research.chalmers.se, 2023-04-26 09:58 UTC

Citation for the original published paper (version of record):

Nuter, R., Korneev, P., Thiele, I. et al (2018). Plasma solenoid driven by a laser beam carrying an orbital angular momentum. Physical Review E, 98(3).

http://dx.doi.org/10.1103/PhysRevE.98.033211

N.B. When citing this work, cite the original published paper. 


\title{
Plasma solenoid driven by a laser beam carrying an orbital angular momentum
}

\author{
R. Nuter, ${ }^{1} \mathrm{Ph}$. Korneev, ${ }^{2,3}$ I. Thiele, ${ }^{4}$ and V. Tikhonchuk ${ }^{1,5}$ \\ ${ }^{1}$ Université Bordeaux, CNRS, CEA, UMR 5107, 33405 Talence, France \\ ${ }^{2}$ National Research Nuclear University “MEPhI”, Moscow 115409, Russian Federation \\ ${ }^{3}$ Lebedev Physical Institute, Moscow 119333, Russian Federation \\ ${ }^{4}$ Department of Physics, Chalmers University of Technology, SE-41296 Göteborg, Sweden \\ ${ }^{5}$ ELI-Beamlines, Institute of Physics, Czech Academy of Sciences, 25241 Dolni Brezany, Czech Republic
}

(Received 10 April 2018; revised manuscript received 5 June 2018; published 26 September 2018)

\begin{abstract}
A megagauss quasistatic axial magnetic field can be produced from the interaction of an intense laser beam carrying an orbital angular momentum with an underdense plasma. Three-dimensional 'particle in cell" simulations and analytical model demonstrate that orbital angular momentum is irreversibly transferred from a tightly focused radially polarized laser beam to electrons without any dissipative effect. A theoretical model describing the individual interaction of electrons with laser shows that particles gain angular momentum during their radial and longitudinal motion in the laser field. The electron rotation and the generated axial magnetic field survive to the end of the laser-plasma interaction and continue over a long time. The agreement between particle in cell simulations and the simplified model identifies routes to increase the intensity of the solenoidal magnetic field by controlling the laser beam characteristics, such as, for example, the orbital angular momentum and/or the pulse duration.
\end{abstract}

DOI: 10.1103/PhysRevE.98.033211

\section{INTRODUCTION}

Magnetic fields are present in nature on different scales. One finds them on extremely large scales of planets and stars in astrophysics [1] to reduced scales in accelerator techniques for the particle beams guiding and focusing [2]. Generating high strength magnetic fields is essential for many projects and research programs, as it is demonstrated by the International Conference on MegaGauss Magnetic field generation.

For less than 20 years, the laser-plasma community has brought a growing contribution to this topic. The generation of a quasistatic long time standing magnetic field in the laser-plasma interaction is a subject of many theoretical [3-13] and experimental studies [14-16]. Two approaches were considered. One consisted in designing the target in such a way that the interaction with the laser generated controlled azimuthal currents $[10,11]$. Another one proposed to transfer angular momentum from laser to electrons by using circularly polarized laser beam $[3-9,14-16]$ or laser beam with a structured spatial shape $[12,13]$. In [3-9], the authors considered theoretically the magnetization of a medium exposed to a circularly polarized Gaussian laser beam. Plasma magnetization originated from the inverse Faraday effect, where the spin angular momentum of a laser beam was transferred to the plasma electrons due to dissipation processes such as collisions, ionization, or radiation friction. This laser to electron angular momentum transfer has been experimentally observed [14-16]. Ali et al. [12], considered a linearly polarized laser beam carrying orbital angular momentum (OAM) [17] and analytically demonstrated that such a laser beam transfers its OAM to electrons through the inverse bremsstrahlung dissipative process. Lécz et al. [13] and Wang et al. [18] numerically modeled the interaction of a screw-shaped laser pulse with an underdense plasma and observed laser to electron OAM transfer in the laser wakefield.

In this article, we present a setup where a quasistatic axial magnetic field is generated through a purely optical process. In contrast to previous works [3-9,12-16], this setup does not need any dissipative process, the electrons gain OAM irreversibly from their driven oscillatory motion in a spatially inhomogeneous laser beam. The axial magnetic field, produced in an underdense plasma irradiated by a radially polarized OAM laser beam, is presented in Sec. II. A technique allowing the emission of such laser beams was described by Li et al. [19], who predicted the efficiency of excitation of the radial component attaining 98\%. In Sec. III, threedimensional (3D) particle in cell (PIC) simulations, modeling the laser-plasma interaction, show an efficient orbital angular momentum transfer from laser to electrons. A laser with intensity equal to $2 \times 10^{18} \mathrm{~W} / \mathrm{cm}^{2}$ generates a megagauss range solenoidal magnetic field. This is a much more efficient process compared to previous works $[4,12]$ where similar magnetic field strengths were expected with higher laser intensities, $7.3 \times 10^{22} \mathrm{~W} / \mathrm{cm}^{2}$ and $10^{19} \mathrm{~W} / \mathrm{cm}^{2}$, respectively. Section IV presents a simplified model describing the laserelectron dynamics. It demonstrates that the laser to electron OAM transfer originates from the joint radial and longitudinal electron motion in the laser field and does not require any dissipative process. The agreement observed between the 3D PIC simulations and the simplified model provides means for controlling the magnetic field with laser parameters. Particularly, the axial B-field amplitude can be strengthened by increasing the laser pulse duration. 


\section{RADIALLY POLARIZED LASER BEAM CARRYING OAM}

\section{A. Input laser beam}

To model a tightly focused radially polarized laser beam carrying OAM, as presented in [19], we consider the numerical algorithm developed by Thiele et al. [20]. It consists in prescribing the temporal and spatial shape for the electromagnetic fields at the focal point $x=x_{0}$ in a vacuum, and then solving the Maxwell's equations in a vacuum to compute the electric and magnetic fields' components at the box boundaries $(x=0$ plane). This method provides the electromagnetic fields consistent with the Maxwell's equations and is valid beyond the paraxial approximation for a laser beam. In our study, the radially polarized OAM laser beam is prescribed at the focal point $x=x_{0}$ in the cylindrical coordinates $(r, \theta, x)$ :

$$
\vec{E}\left(r, \theta, t, x_{0}\right)=E_{0} g(t) f(r) \cos \left(l \theta-\omega_{0} t\right) \vec{e}_{r},
$$

with the radial distribution $f(r)=C_{l}\left(r / w_{0}\right)^{|l|} e^{-\left(r / w_{0}\right)^{2}}$, the temporal envelope $g(t)=\cos ^{2}\left(\pi \frac{t-t_{0}}{\tau}\right)$ in the time interval $\left|t-t_{0}\right|<\tau / 2$, the laser OAM $l$, the focal beam waist $w_{0}=2 \mu \mathrm{m}$ (2.5 times the laser wavelengths), the pulse duration $\tau$ equal to six optical periods $\left(\tau_{0}\right)$, the central time $t_{0}$, the laser amplitude $E_{0}$, the normalization factor $C_{l}=\sqrt{2^{|l|+1} /|l| !}$, and the radial unit vector $\vec{e}_{r}$. The laser frequency $\omega_{0}=2.3 \times 10^{15} s^{-1}$ corresponds to the laser wavelength $=0.8 \mu \mathrm{m}$. The electromagnetic energy distribution for the $l=1$ laser beam is illustrated in Fig. 1. It is characterized by two entangled helices with electric field vectors directed along the same radial direction.

The components of such a laser beam at the numerical box left border are computed by using the numerical algorithm described in [20]. Then its longitudinal propagation in a vacuum or in plasma is computed with the numerical Maxwell solver implemented in the PIC code OCEAN.

\section{B. Analytical expressions}

By using the Maxwell-Gauss and the Maxwell-Faraday equations, we compute the longitudinal component of the electric field and the magnetic fields' components at the focal point. The electric and magnetic field components are written at $x=x_{0}$ :

$$
E_{r}=a_{0} f(r) g(t) \cos (\phi), \quad B_{r}=0,
$$

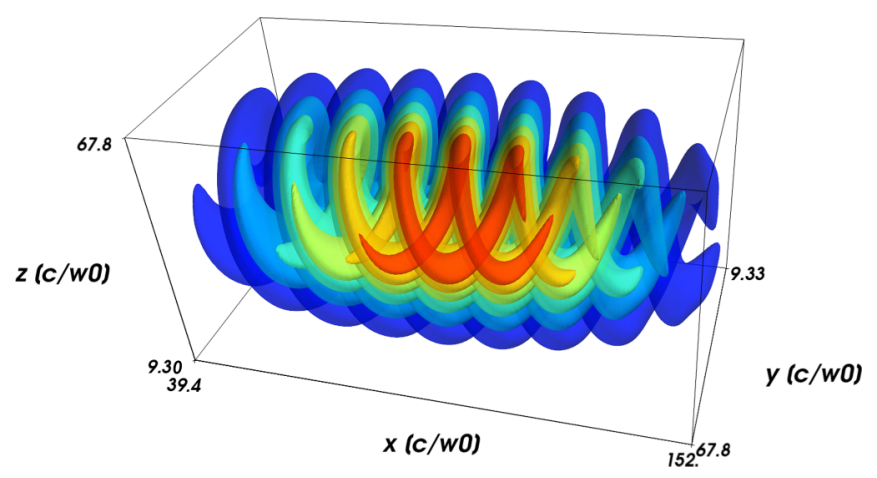

FIG. 1. An OAM $l=1$ laser beam helical energy distribution.

$$
\begin{gathered}
E_{\theta}=0, \quad B_{\theta}=a_{0} f(r) g(t) \cos (\phi) \\
E_{x}=a_{0} \frac{f(r)}{r} g(t) \sin (\phi), \quad B_{x}=-l a_{0} \frac{f(r)}{r} g(t) \cos (\phi),
\end{gathered}
$$

where $\phi=\omega_{0} t-l \theta-k_{0} x$ is the phase term, $k_{0}$ is the wave number, and $a_{0}=e E_{0} / m_{e} \omega_{0} c$ ( $m_{e}$ is the electron mass, $e$ is the electron charge, and $c$ is the light speed in a vacuum) is the dimensionless field amplitude.

Figure 2 displays the transverse cut of the electric field components at the focal point, $x_{0}=46 \mathrm{c} / \omega_{0}$, for $a_{0}=1$, $l=1$, and $\omega_{0}=2 \mu \mathrm{m}$. As expected, the radial component dominates. Its transverse cut presents two lobes containing an electric field oriented along the same direction. A small longitudinal component, dephased with the radial component by a factor $\pi / 2$, appears in agreement with previous equations. The azimuthal component is zero.

Similar electric field components are presented in Fig. 3, but at a longitudinal position localized before the focal point, $x=30 c / \omega_{0}$, only three laser wavelengths from the focus.

We still observe two lobes for the radial and longitudinal electric fields, but this time the field azimuthal component is nonzero. It contains also two lobes nearly phase-matched with the radial component, but a smaller amplitude. This nonzero azimuthal component appears out of the focal point because the radially polarized laser beam prescribed in Eq. (1) is not the eigensolution of the Maxwell equations. The Laguerre Gauss (LG) beams are known to verify the wave equation in the paraxial approximation [17,21], with a linear polarization. For our study, the laser beam is a LG-like beam with a radial polarization, where the polarization vector $\vec{e}_{r}=\cos \theta \vec{e}_{y}+$ $\sin \theta \vec{e}_{z}$ depends on the azimuthal angle $\left(e_{y}, e_{z}\right.$ are the unit vectors in the transverse plane). This analytical expression is not an eigensolution of the Maxwell equations. Moreover, we consider a strongly focused laser beam so that the paraxial approximation is no more valid to model the laser beam. As a result, the electromagnetic fields do not propagate by keeping the spatial characteristics described in Eqs. (2), (3), and (4); a nonzero azimuthal component appears out of the focal point. Figures 3 and 4 show that this azimuthal component can reach at maximum $20 \%$ of the radial electric field amplitude. It varies along the propagation direction. Its amplitude is reduced down to zero at the focal point, and then, after the focal point, is increased from zero value up to $20 \%$ of the radial electric field amplitude.

We do not have access to the analytical expressions for the electromagnetic fields defining this radially polarized LG beam. However, their numerical expressions can be computed from the Maxwell solver developed in the PIC code OCEAN and from the numerical algorithm developed by Thiele et al. [20]. Both numerical solutions give the same electromagnetic patterns which can be approximated by

$$
E_{r}=a_{0} f(r) g(t) \cos (\phi), \quad B_{r}=-l a_{0} \alpha f(r) g(t) \cos (\phi),
$$

$E_{\theta}=l a_{0} \alpha f(r) g(t) \cos (\phi), \quad B_{\theta}=a_{0} f(r) g(t) \cos (\phi)$, 

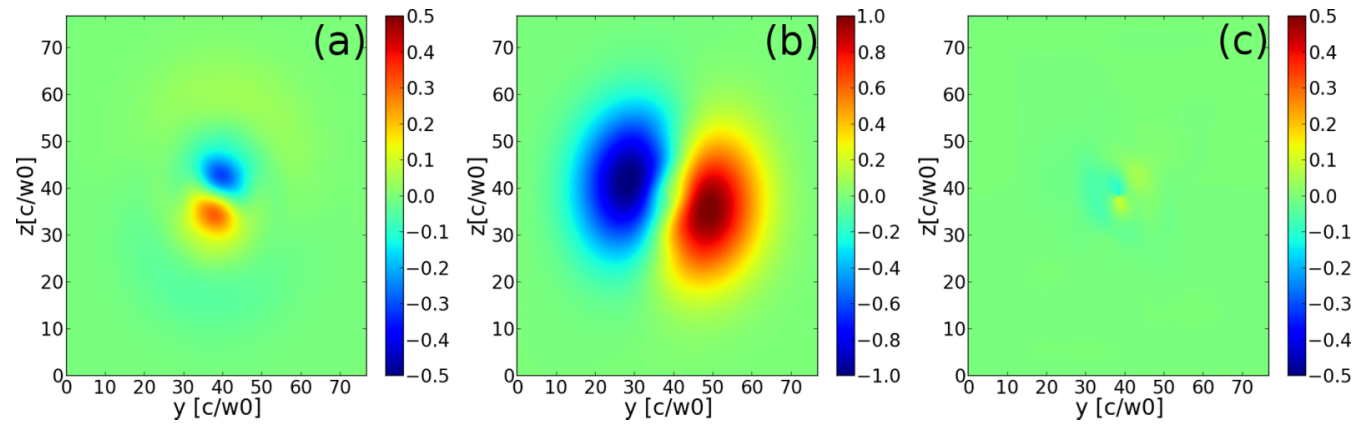

FIG. 2. (a) Longitudinal, (b) radial, and (c) azimuthal components of the laser electric field computed at the focal point $x_{0}=46 c / \omega_{0}$ in PIC simulations with no plasma.

$$
E_{x}=a_{0} \frac{f(r)}{r} g(t) \sin (\phi), \quad B_{x}=-l a_{0} \frac{f(r)}{r} g(t) \cos (\phi),
$$

where $\alpha=0.1$ models an averaged variation of the ratio between the azimuthal and the radial field component along the propagation direction.

\section{MAGNETIC FIELD GENERATION}

\section{A. Particle in cell simulations}

Interaction of such a laser beam with a collisionless underdense plasma is studied with the 3D PIC code OCEAN [22]. The numerical box is composed of 640 cells along the longitudinal axis $x$, and $512 \times 512$ cells in the transverse plane $(y, z)$, with a spatial resolution of $19 \mathrm{~nm}$. Absorbing conditions for the electromagnetic fields and particles are defined at the box boundaries. The laser pulse with an OAM $l=1$ and an intensity $2 \times 10^{18} \mathrm{~W} / \mathrm{cm}^{2}$, corresponding to the dimensionless field amplitude $a_{0}=1$, is injected into the numerical box from the left border. The total laser energy is $2.5 \mathrm{~mJ}$. The plasma composed of electrons and protons has an initial density equal to $1.74 \times 10^{19} \mathrm{~cm}^{-3}$, corresponding to $1 \%$ of the plasma critical density $n_{c}=1.74 \times 10^{21} \mathrm{~cm}^{-3}$. The plasma has a cylindrical shape with a $1.52 \mu \mathrm{m}$ length and a $6.9 \mu \mathrm{m}$ diameter. These small plasma dimensions have been chosen to reduce the CPU time of 3D PIC simulations. However, it is verified that the presented results hold for any plasma geometry provided that the plasma transverse size is larger than the laser beam waist. Ten macroparticles per cell are considered.

In PIC simulations, we compute the average longitudinal orbital angular momentum gained by electrons in the laser beam

$$
\left\langle L_{x}\right\rangle=\frac{1}{N_{b}} \sum_{i=1, N_{b}} w_{i}\left[\left(y_{i}-y_{0}\right) \times p_{z, i}-\left(z_{i}-z_{0}\right) \times p_{y, i}\right]
$$

with the total number of "electron" macroparticles $N_{b}$, laser beam axis $\left(y_{0}, z_{0}\right)$, transverse coordinates $\left(y_{i}, z_{i}\right)$, and transverse momenta $\left(p_{y, i}, p_{z, i}\right)$ of the macroparticle $i$ defined with the weight $w_{i}$. Protons do not gain any significant angular momentum due to their mass 1836 times heavier than the electron's one. The solid curves in Fig. 5 display the temporal evolution of $\left\langle L_{x}\right\rangle$ : the red and green curves, distinguishing electrons rotating in opposite directions, show a similar temporal behavior but of an opposite sign. As the laser interacts with the plasma bulk, from $\omega_{0} t=50$ to $\omega_{0} t=100\left(1 / \omega_{0}=0.4248 \mathrm{fs}\right)$, the absolute value of $\left\langle L_{x}\right\rangle$ increases up to $0.5 m_{e} c^{2} / \omega_{0}$, and then decreases to a value close to $0.15-0.2 m_{e} c^{2} / \omega_{0}$. The black curve, displaying the total average electron angular momentum, exhibits a break around $\omega_{0} t \simeq 70$. $\left\langle L_{x}\right\rangle$ remains nonzero once the electronlaser interaction ends: equal to $-0.08 m_{e} c^{2} / \omega_{0}$ at $\omega_{0} t \simeq 75$, it slightly increases up to $-0.063 m_{e} c^{2} / \omega_{0}$ at $\omega_{0} t \simeq 250$. The dashed curves in Fig. 5, displaying the relative number of electrons with positive and negative angular momentum, show that the part of electrons with negative angular momentum continuously increases during their interaction with the laser.
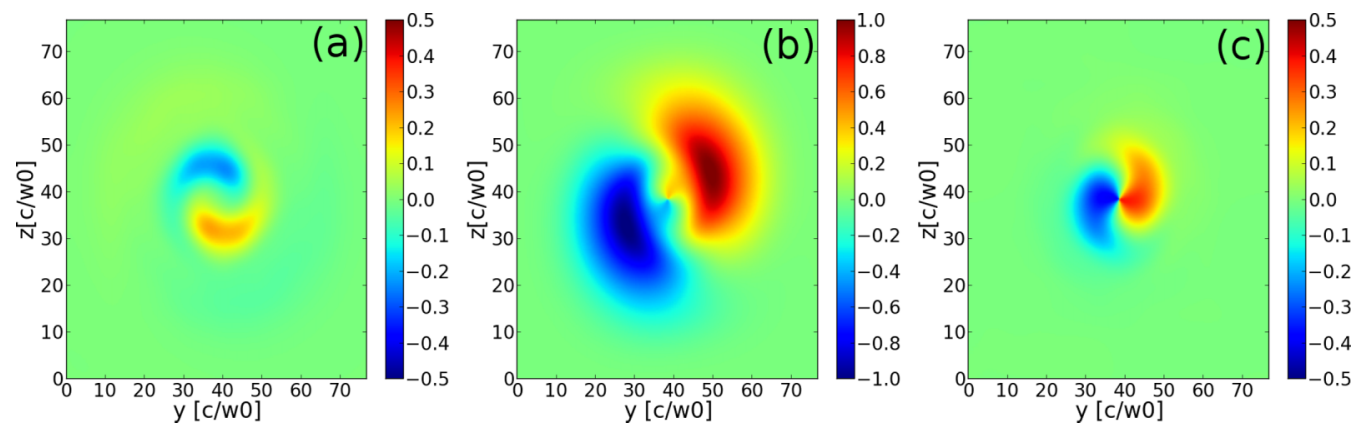

FIG. 3. (a) Longitudinal, (b) radial, and (c) azimuthal components of the laser electric field computed before the focal point at $x=30 c / \omega_{0}$ in the PIC simulations with no plasma. 

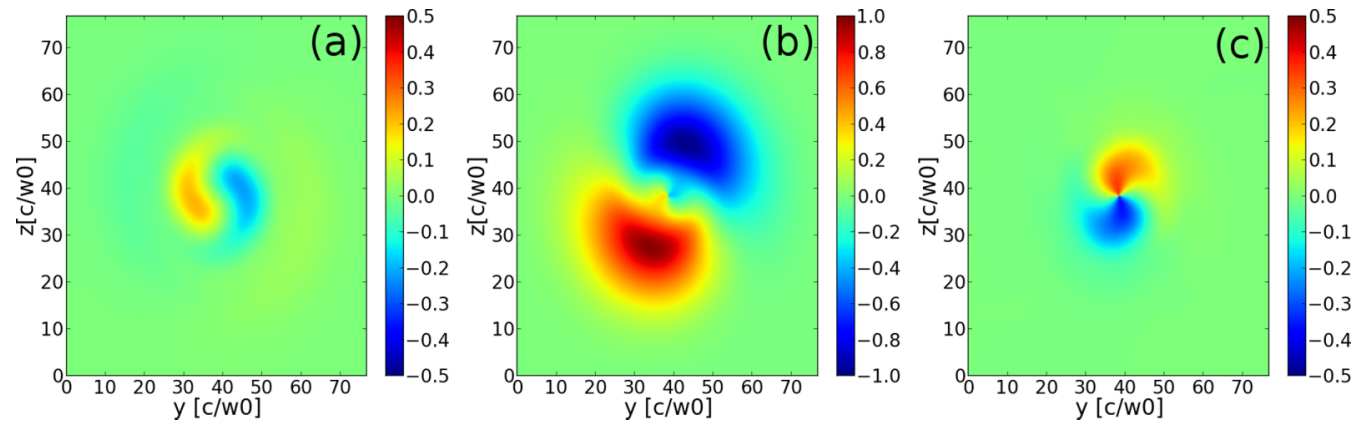

FIG. 4. (a) Longitudinal, (b) radial, and (c) azimuthal components of the laser electric field computed after the focal point at $x=63 c / \omega_{0}$ in the PIC simulations with no plasma.

At the end of their interaction with the laser, more than $70 \%$ of electrons have acquired a negative angular momentum.

The transversal cut of the electron longitudinal OAM distribution, shown in Fig. 6, displays a ring (a tube in 3D) containing the highest electron $L_{x}$ amplitude with an external radius $r_{1} \sim 15 \mathrm{c} / \omega_{0}$ and an internal one $r_{0} \sim 6 \mathrm{c} / \omega_{0}$. These rotating electrons, characterized by $L_{x}$ slightly lower than $-0.4 m_{e} c^{2} / \omega_{0}$, form a solenoidal structure with an axial magnetic field inside (see Fig. 7) The central hole shows $L_{x}$ close to zero, in agreement with the laser intensity distribution.

Figure 7 displays an isocontour of the longitudinal magnetic field $B_{x}$ once the laser has left the plasma area. The quasistatic $B_{x}$ field presents a cylindrical shape: its axial length is equal to the plasma length $(\sim 2.5 \mu \mathrm{m})$ and its radius is limited by the contour where the laser intensity is maximal $\left(r=12 c / \omega_{0}\right)$. The cylinder axis coincides with the laser one. The $B_{x}$ maximal amplitude reaches $0.004 B_{c}$, where $B_{c}=m_{e} \omega_{0} / e=13382 \mathrm{~T}$. In the International System of units, this corresponds to $53 \mathrm{~T}$ magnetic field, i.e., 0.5 megagauss.

\section{B. Solenoid model}

We compare this value to the $B_{x}$ field generated by electrons localized in a hollow cylinder with a length $d$, an internal radius $r_{0}$, and an external radius $r_{1}$. The electron density is set

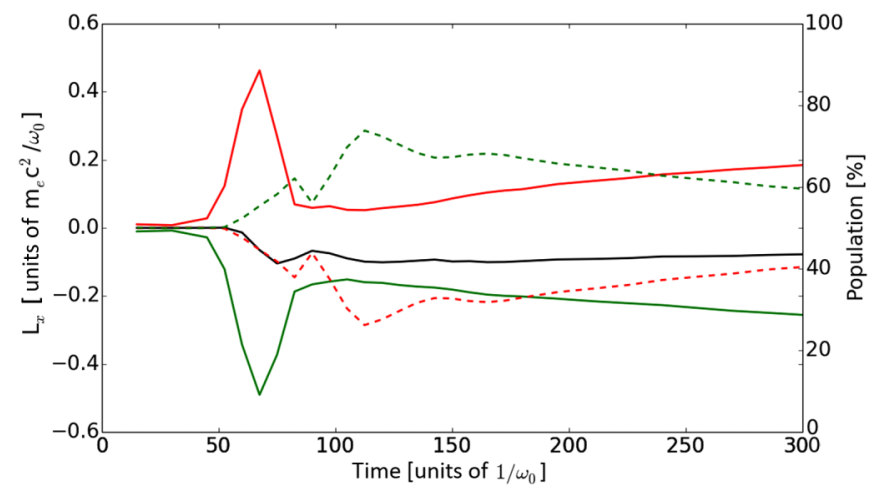

FIG. 5. Longitudinal orbital angular momentum averaged over all the electron population (black solid curve), over electrons with positive $L_{x}$ (red solid curve), and negative $L_{x}$ (green solid curve). Fraction of electron population with positive (red dashed curve) and negative (green dashed curve) $L_{x}$. to $n_{e}$ and the electron longitudinal orbital angular momentum is equal to $l_{x}$. We consider the laser unit system, where lengths are expressed in $c / \omega_{0}=127.3 \mathrm{~nm}$, density is expressed in $n_{c}$ and magnetic field is expressed in $B_{c}$. The derivation of the Biot-Savart law [23], presented in Appendix A results in

$$
B_{x} \simeq-0.5 n_{e} l_{x}\left[\frac{d}{r_{0}}-\frac{d}{r_{1}}\right]
$$

We consider the values computed in PIC simulations: $l_{x}=\left\langle L_{x}\right\rangle=-0.4 m_{e} c^{2} / \omega_{0}, n_{e}=0.01 n_{c}, d=19 c / \omega_{0}, r_{0}=$ $6 c / \omega_{0}$, and $r_{1}=15 c / \omega_{0}$. Note that due to the longitudinal ponderomotive force experienced by the electrons, the plasma has been longitudinally expanded from its initial size $d=11.9 \mathrm{c} / \omega_{0}$ to $d=19 \mathrm{c} / \omega_{0}$. By applying these numerical values to Eq. (9), we obtain $B_{x}=0.0038 B_{c}$ which perfectly agrees with the $B_{x}$ value computed from PIC simulations and equal to $0.004 B_{c}$. This perfect agreement confirms that the magnetic field observed in the PIC simulations originates from the efficient electron OAM gain.

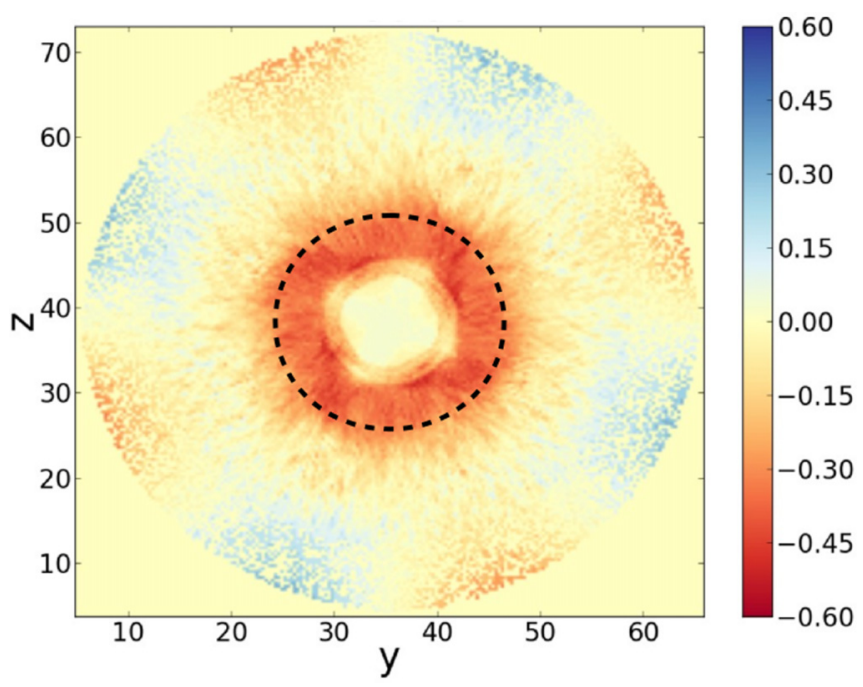

FIG. 6. Transversal cut of the electron $L_{x}$ distribution near $x=$ $30 \mathrm{c} / \omega_{0}$ for $\omega_{0} t=120$. The dashed black curve displays the field maximum amplitude contour. 


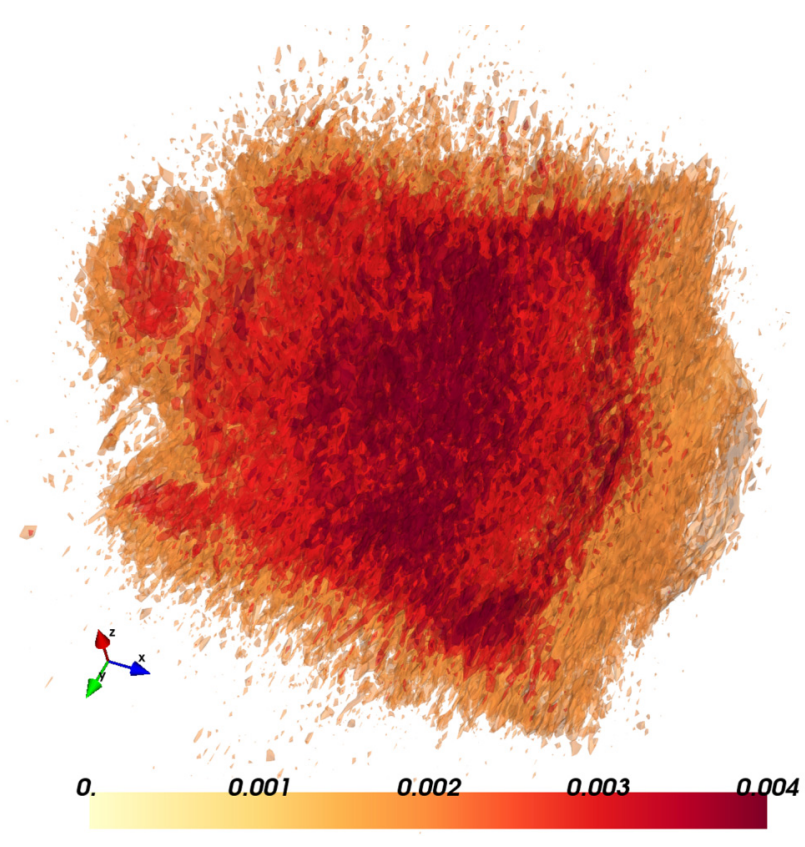

FIG. 7. Solenoidal magnetic field $B_{x}$ for $\omega_{0} t=120$.

\section{LASER TO ELECTRON OAM TRANSFER}

\section{A. Electron tracking in simulations}

To understand how electrons gain their angular velocity, we implemented in the OCEAN code a particle tracking module. This diagnostic consists in adding some arbitrarly chosen electrons that experience the electric and magnetic fields but do not produce the self-consistent fields, automatically computed in PIC simulations. Figure 8 displays the longitudinal OAM acquired by these "test electrons" at the end of their interaction with the laser beam as a function of their initial radial coordinate $\left(r_{0}\right)$, when the plasma motion is active (green circle) so that plasma self-consistent fields influence the laserelectron dynamics and when the plasma motion is "frozen" (gray markers) where these "test electrons" only experience the laser electromagnetic fields.

The green circles show that $L_{x}$ values lower than $-0.4 m_{e} c^{2} / \omega_{0}$ are obtained for electrons initially localized in the zone where the laser intensity is maximum, i.e., $r=7-12 c / \omega_{0}$. Moreover, the $L_{x}$ spatial distribution follows the laser intensity spatial shape. Same characteristics are observed for the $L_{x}$ radial distribution when the plasma motion is frozen (gray markers), except that the electron angular momenta reach higher absolute values. This demonstrates that the OAM transfer from laser beam to each electron is a pure optical process for which the efficiency increases as the plasma self-consistent fields are turned off. When the plasma motion is active, a longitudinal charge separation field is generated on the rear plasma surface due to the ion inertia. This electrostatic field keeps the electrons confined in the plasma zone reducing their interaction time with the laser, and then their angular velocity gain. To overcome this limitation, the plasma length should be increased.

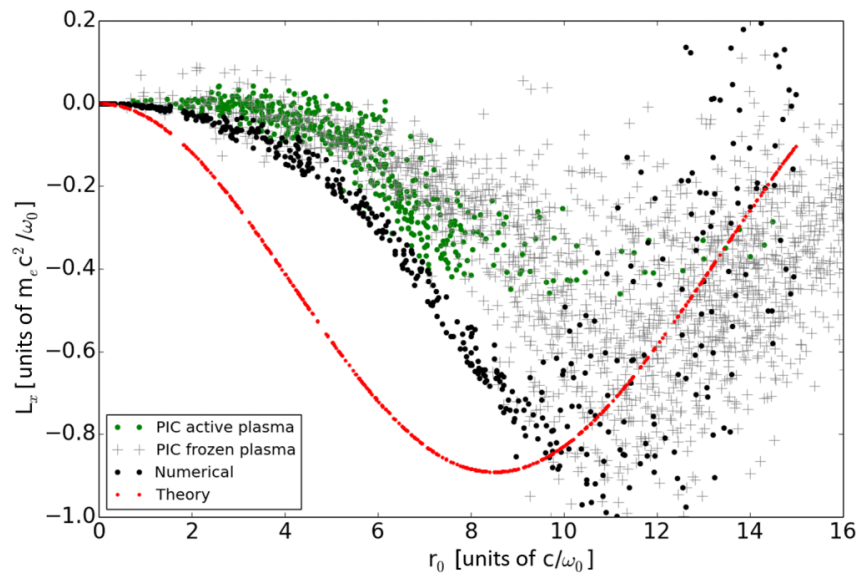

FIG. 8. Longitudinal orbital angular momentum $\left(L_{x}\right)$ computed at $\omega_{0} t=97$ versus the initial electron radial position with (green circle) and without (gray markers) active plasma motion. The red dotted curve and the black circles display the $L_{x}$ values computed, analytically by using the perturbation theory and numerically, respectively.

\section{B. Reduced model and the perturbative development}

The PIC simulations demonstrate that the laser to electrons OAM transfer results from a nondissipative optical process. We confirm it by developing a reduced numerical model describing the laser beam induced electron dynamics. It solves the relativistic equations of electron motion in the electric $\left(E_{r}\right.$, $\left.E_{\theta}, E_{x}\right)$ and magnetic fields $\left(B_{r}, B_{\theta}, B_{x}\right)$ of which analytical expressions are given in Eqs. (5), (6), and (7)

$$
\begin{gathered}
\dot{p_{r}}=-E_{r}-v_{\theta} B_{x}+v_{x} B_{\theta}+p_{\theta} \dot{\theta}, \\
\dot{p_{\theta}}=-E_{\theta}-v_{x} B_{r}+v_{r} B_{x}-p_{r} \dot{\theta}, \\
\dot{p}_{x}=-E_{x}-v_{r} B_{\theta}+v_{\theta} B_{r},
\end{gathered}
$$

where $p_{r}=\gamma v_{r}=\gamma \dot{r}, p_{\theta}=\gamma v_{\theta}=\gamma r \dot{\theta}$, and $p_{x}=\gamma v_{x}=\dot{x}$ are the radial, azimuthal, and longitudinal electron momenta, respectively, and $\gamma=\sqrt{1+p_{r}^{2}+p_{\theta}^{2}+p_{x}^{2}}$ is the Lorentz factor.

First, we assume a low laser intensity $\left(a_{0}<<1\right)$ which allows a perturbative expansion of Eqs. (10), (11), and (12) in powers of $a_{0}$. The details on the analytical computations are given in Appendix B. Integrated over the pulse duration, the first order of electron OAM is zero, so that it does not contribute to the final electron OAM. The second-order term of $L_{x}$ solves

$$
{\dot{L_{x}}}^{(2)}=r^{(1)} \dot{p}_{\theta}^{(1)}-r_{0} p_{x}^{(1)} B_{r}+r_{0} p_{r}^{(1)} B_{x}-r_{0} \frac{d E_{\theta}}{d \vec{r}}\left(\vec{r}^{(1)}-\vec{r}_{0}\right) .
$$

Only the $-r_{0} \frac{d E_{\theta}}{d r}\left(r^{(1)}-r_{0}\right)$ and $-r_{0} \frac{d E_{\theta}}{d x}\left(x^{(1)}-x_{0}\right)$ terms finally contribute to

$$
L_{x}^{(2)}(\tau)=-\alpha l a_{0}^{2} f^{2}\left(r_{0}\right) \frac{3 \tau}{8}\left[1-\left(\frac{r_{0}}{w_{0}}\right)^{2}\right] .
$$




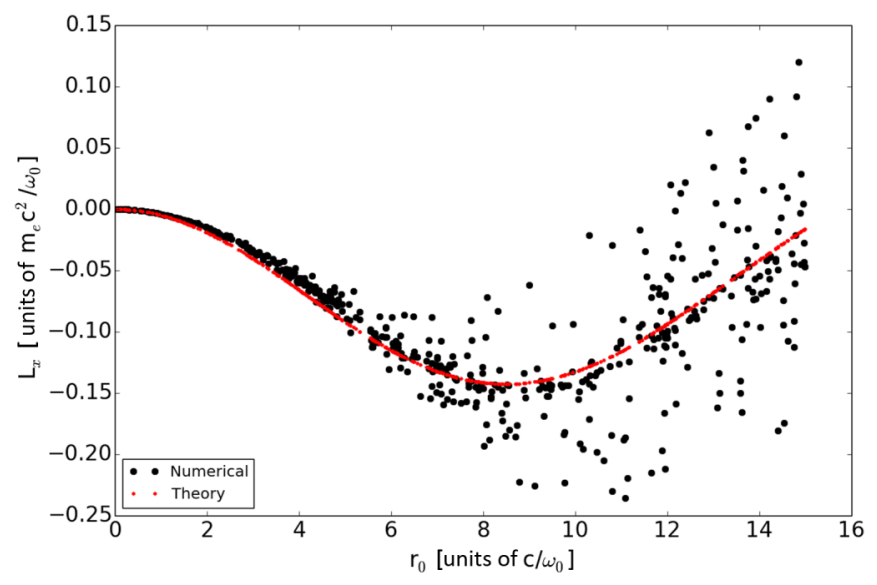

FIG. 9. Longitudinal orbital angular momentum of electrons versus their initial radial position computed from the numerical integration of motion equations (black circle) and the theoretical prediction according to Eq. (14) (red curve) for $a_{0}=0.4$ and $\alpha=0.1$. The other laser parameters are identical to the PIC simulations: $w_{0}=2 \mu \mathrm{m}$, $\tau=6 \tau_{0}, l=1$.

Figure 9 compares the electron $L_{x}$ radial distribution computed from the numerical integration of Eqs. (10), (11), and (12) (black circle) and from the analytical expression [Eq. (14)] (red curve) for $a_{0}=0.4$. We note a perfect agreement between both curves. As already observed in the PIC simulations (Fig. 8), even at low intensity, most of the electrons acquire a negative longitudinal OAM. This analysis evidences that the laser to electron OAM transfer originates from the radial and longitudinal electron drift in the laser field. For $r_{0}=w_{0} / \sqrt{2}$ (where the intensity is maximum), the electrons acquire the maximum angular rotation, and the theoretical development of $L_{x}^{(2)}$ (not shown here) enables to quantify that the electron longitudinal motion contributes for $2 / 3$ and the radial one for $1 / 3$ to the final angular momentum value.

For higher laser intensity, $a_{0}=1$, the black circles and the red curve in Fig. 8 display the $L_{x}^{(2)}$ values computed by numerically integrating Eqs. (10), (11), and (12) and with the theoretical formula [Eq. (14)], respectively. The analytical expression deviates from the numerical integration of motion equations, but demonstrates a qualitatively similar behavior. We observe negative longitudinal OAM gained by electrons, and the correct order of magnitude for the $L_{x}$ values. A relatively good agreement between numerical results and PIC simulations is evident even though the azimuthal component of the field is chosen constant along the laser propagation in the numerical model. In both cases, the laser transfers to electrons negative $L_{x}$ values, and the OAM transfer efficiency follows the laser intensity radial distribution.

\section{Control of the magnetic field strength}

Equation (14) predicts that the sign of the electron OAM values should change by inverting the sign of the OAM laser beam. This is confirmed in Fig. 10 where the $L_{x}$ spatial distribution computed for $a_{0}=1$ and $l=-1$ displays positive values. Note that, similarly to the $l=1$ case, a discrepancy is observed between the theoretical perturbative expression and the

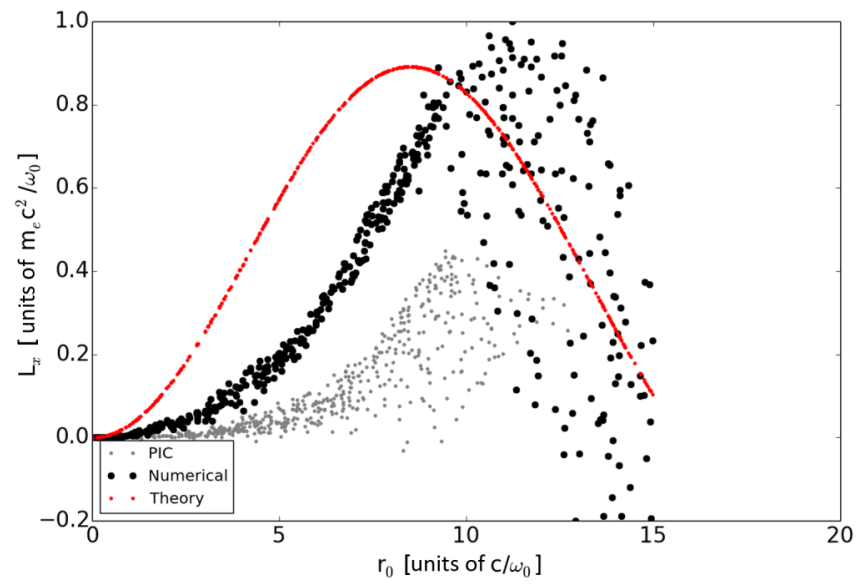

FIG. 10. $L_{x}$ versus the electron initial radial position computed with PIC simulations with active plasma (gray dot), with the numerical resolution of motion equations (black circle) and with the perturbative formula given in Eq. (14) (red curve) for $a_{0}=1$ and $l=-1, \tau=6 \tau_{0}, w_{0}=2 \mu \mathrm{m}$.

numerical values of $L_{x}$. This originates from the limit of the perturbative theory which disregards some kinetic processes of higher orders, like, for example, the ponderomotive force that increases the longitudinal displacement of the electrons. Despite this discrepancy, both curves show positive electron longitudinal OAM values, opposite to the laser OAM value. Moreover, we note a relative good agreement between the numerical integration of motion equations (black circles) and PIC simulations (gray dots). Once again, the PIC computed $L_{x}$ values are smaller because of the plasma induced selfconsistent fields which reduce the time interaction between electrons and laser.

By controlling the electron OAM values with the laser OAM values, we choose the direction of the solenoidal magnetic field direction. Figure 11(a) shows the longitudinal cut of the solenoid magnetic field generated with a laser beam carrying $l=-1$ OAM. The $B_{x}$ amplitude is equal to the one computed for $l=1$, but now the solenoid $B$ field is oriented along the decreasing $x$ values.

Equation (14) predicts also that the electron OAM amplitude is higher as the pulse duration increases. Figure 11(b), displaying the longitudinal cut of $B_{x}$ computed to a pulse duration equal to 12 optical cycles, shows that its amplitude is two times higher than for a pulse duration equal to 6 optical cycles; the axial magnetic field reaches $100 \mathrm{~T}$. The longitudinal extension of the magnetic field is larger than for the shorter pulse duration because the electrons have acquired higher longitudinal momenta resulting in a larger plasma expansion.

These results clearly evidence that this laser-plasma interaction setup not only can generate a quasistatic magnetic field, but also enable a perfect control of its generation.

\section{CONCLUSION}

In conclusion, we demonstrate that a tightly focused radially polarized laser beam is able to transfer its orbital angular momentum to electrons without any dissipative effects like 

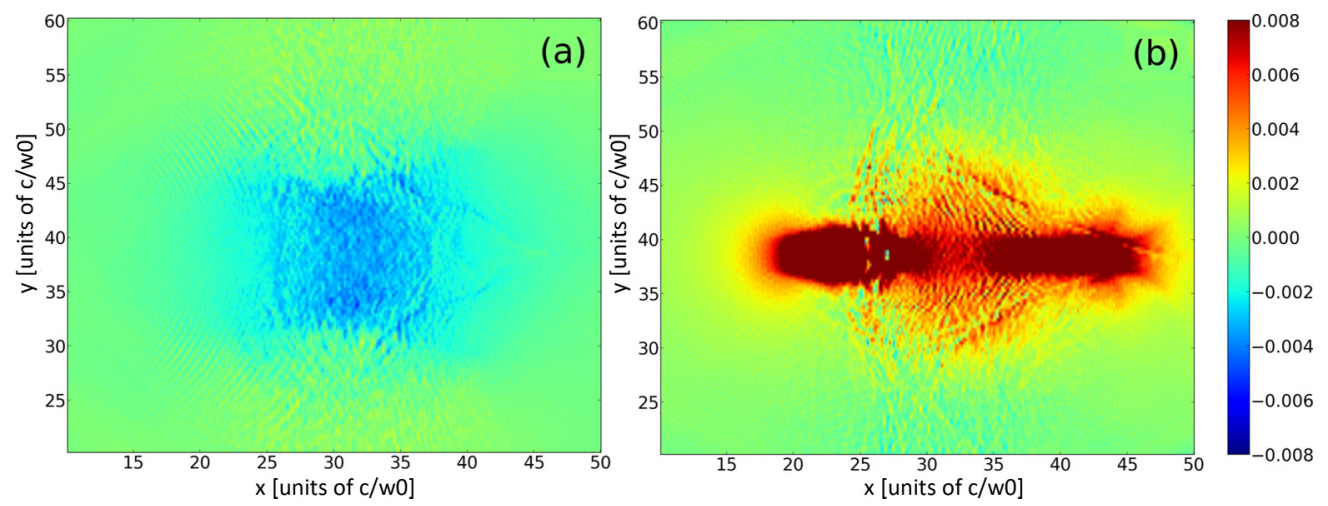

FIG. 11. Longitudinal cut of $B_{x}$ computed from PIC simulations for (a) $l=-1$ and $\tau=6 \tau_{0}$ and (b) $l=1$ and $\tau=12 \tau_{0}$ five optical periods after the laser-electron interaction ends. The others laser parameters are kept unchanged.

collision or ionization. A nondissipative optical process is responsible for the irreversible electron rotation secular gain, which originates from the radial and longitudinal electron drift in the laser field. Both radial and azimuthal components of the laser fields are needed for the laser to electron OAM transfer. By using 3D particle in cell simulations, we show that the rotating electrons produce a quasistatic magnetic field of megagauss range over more than $80 \mathrm{fs}$ after the end of the laser-plasma interaction. The lifetime is limited by the plasma expansion due to the laser-induced ponderomotive force. This magnetic field is homogeneous over spatial dimensions (a $2.5 \mu \mathrm{m}$ length and a $1.5 \mu \mathrm{m}$ as transverse size) defined by the plasma length and the laser transverse size. An accurate choice of laser parameters, such as focal beam waist, laser intensity, laser OAM, and/or pulse duration, make then possible a control of the quasistatic magnetic field production. A simple analytical model provides a quantitative estimate of the electron longitudinal orbital angular momentum values and the solenoidal magnetic field up to $a_{0}=1$. This work opens alternative ways to optically generate megagauss quasistatic magnetic fields from plasma interacting with mJ laser beam. We can expect a gigagauss axial B-field from a laser beam in a Joule range.

\section{ACKNOWLEDGMENTS}

This work was granted access to HPC resources of TGCC under the allocation A0010506129 made by GENCI and under the allocation 2017174175 made by PRACE. The authors acknowledge support from MEPhI Academic Excellence Project (Contract No. 02.a03.21.0005-27.08.2013) and from the project ELITAS (ELI Tools for Advanced Simulation) CZ.02.1.01/0.0/0.0/16_013/0001793 from the European Regional Development Fund.

\section{APPENDIX A: QUASISTATIC MAGNETIC FIELD}

We detail here the computation of the quasistatic magnetic field produced from an electron solenoid. The solenoid shape is a hollow cylinder with a length $d$, an internal radius $r_{0}$, and an external radius $r_{1}$, as drawn in Fig. 12. The electrons localized in the hollow cylinder are characterized with a density $n_{e}$ and with an longitudinal orbital angular momentum equal to $l_{x}$.
According to the Biot-Savart law [22], the magnetic field generated at the point $M$ from electron localized at the point $P$ is

$$
\vec{B}(M)=\frac{\mu_{0}}{4 \pi} \int_{V} \frac{\vec{j}(P) \times \overrightarrow{P M}}{\|\overrightarrow{P M}\|^{3}} d V,
$$

with $\vec{j}(P)$ the electron density current at the point $P$.

Due to the cylindrical symmetry, the magnetic field is oriented along the $x$ axis, so that the point $M$ is localized on the $x$ axis $\left(\overrightarrow{O M}=x \vec{e}_{x}\right)$. The electrons are localized the point $P$ such that $\overrightarrow{O P}=r \vec{e}_{r}$. We then write $\vec{j}(P) \times \overrightarrow{P M}=$ $-e n_{e} \vec{v}_{\theta} \times\left(-r \vec{e}_{r}+x \overrightarrow{e_{x}}\right)$, where $\vec{v}_{\theta}$ is the electron azimuthal velocity. The axial magnetic field then writes

$$
B_{x}(M)=-\frac{e n_{e} \mu_{0}}{2 m_{e}} l_{x} \int_{0}^{d} d x \int_{r_{0}}^{r_{1}} \frac{r}{\left(r^{2}+x^{2}\right)^{3 / 2}} d r .
$$

After spatial integration, the magnetic field writes:

$$
B_{x}=-0.5 \mu_{0} \frac{e n_{e}}{m_{e}} l_{x}\left[\sinh ^{-1}\left(\frac{d}{r_{0}}\right)-\sinh ^{-1}\left(\frac{d}{r_{1}}\right)\right] .
$$

Expressed in units of $B_{c}=m_{e} \omega_{0} / e$ and in the limit $d \leqslant r_{0}$, $d \leqslant r_{1}$, the axial magnetic field is approximated by Eq. (9).

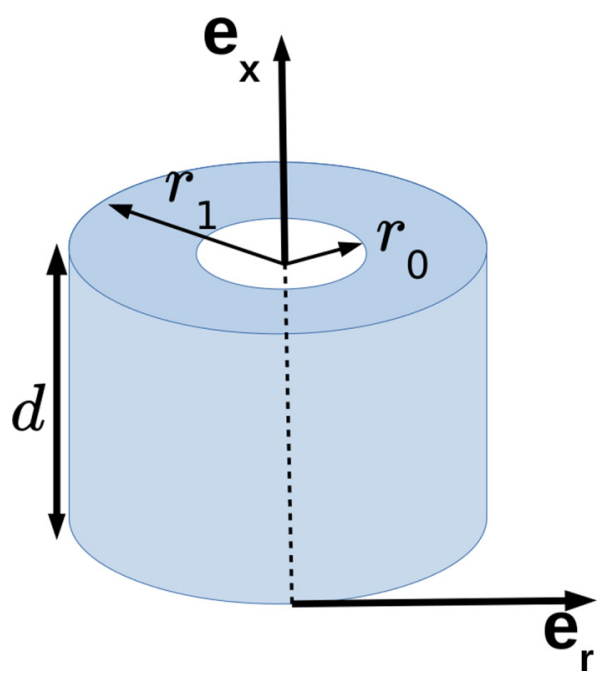

FIG. 12. The electron solenoid with a length $d$, an internal radius $r_{0}$, and an external radius $r_{1}$. Electrons are localized in the blue zone. 


\section{APPENDIX B: PERTURBATIVE TREATMENT}

The theoretical analysis is performed in the laser units system where the electric field, the magnetic field, the momentum, the velocity, the distance, and the time are normalized to $E_{c}=m_{e} \omega_{0} c / e, B_{c}=m_{e} \omega_{0} / e, m_{e} c, c, c / \omega_{0}$, and $1 / \omega_{0}$, respectively, with $c$ the light speed in a vacuum. The perturbative development of the electron motion equations, Eqs. (10), (11), and (12), is performed in the low laser intensity regime, $a_{0} \ll 1$ and $\gamma=1$. of $a_{0}$

The physical quantities are developed in a power series

$$
X_{j}=\sum_{i=0}^{N} a_{0}^{i} X_{j}^{(i)},
$$

where $X_{j}=r, \theta, x, p_{r}, p_{\theta}, p_{x}, L_{x}$ are the cylindrical coordinates, the associated electron momenta, and the electron longitudinal orbital angular momentum. Inserting these developments in Eqs. (10), (11), and (12) and assuming that the laser pulse duration contains a large number of optical cycles, we obtain the first-order terms

$$
\begin{aligned}
r^{(1)} & \simeq a_{0} f\left(r_{0}\right) g(t) \cos \left(t-\phi_{0}\right), \\
\theta^{(1)} & \simeq \alpha a_{0} l \frac{f\left(r_{0}\right)}{r_{0}} g(t) \cos \left(t-\phi_{0}\right), \\
x^{(1)} & \simeq a_{0} \frac{f\left(r_{0}\right)}{r_{0}} g(t) \sin \left(t-\phi_{0}\right), \\
p_{r}^{(1)} & \simeq-a_{0} f\left(r_{0}\right) g(t) \sin \left(t-\phi_{0}\right), \\
p_{\theta}^{(1)} & \simeq-\alpha l a_{0} f\left(r_{0}\right) g(t) \sin \left(t-\phi_{0}\right), \\
p_{x}^{(1)} & \simeq a_{0} \frac{f\left(r_{0}\right)}{r_{0}} g(t) \cos \left(t-\phi_{0}\right),
\end{aligned}
$$

where $r_{0}=r^{(0)}$ and $\phi_{0}=l \theta^{(0)}-x^{(0)}$. The time derivative of the electron angular momentum verifies $\dot{L}_{x}=\dot{r} p_{\theta}+r \dot{p}_{\theta}$. Because, the initial electron angular velocity is zero, the $L_{x}$

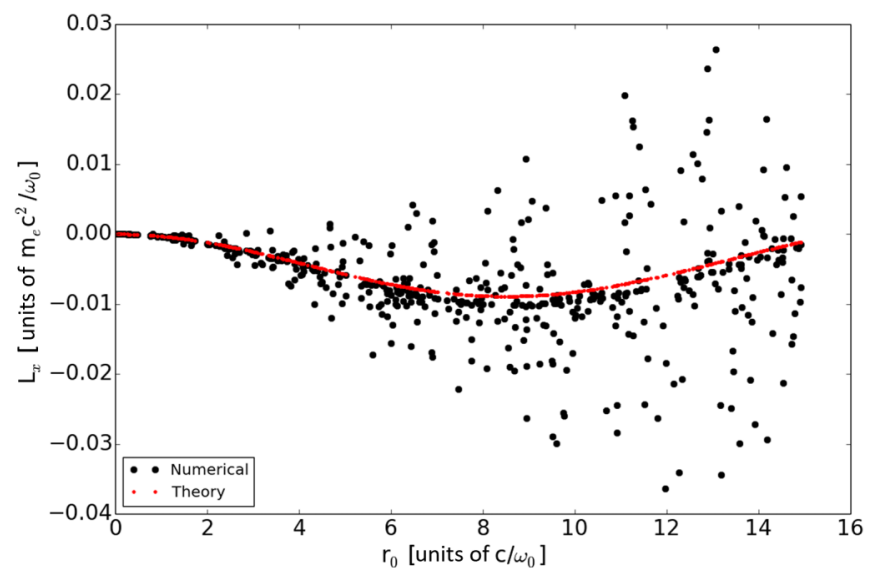

FIG. 13. $L_{x}$ versus the electron initial radial position computed with the numerical integration of motion equations (black circle) and with the perturbative formula given in Eq. (14) (red curve) for $a_{0}=$ 0.1 and $l=1, \tau=6 \tau_{0}, w_{0}=2 \mu \mathrm{m}$.

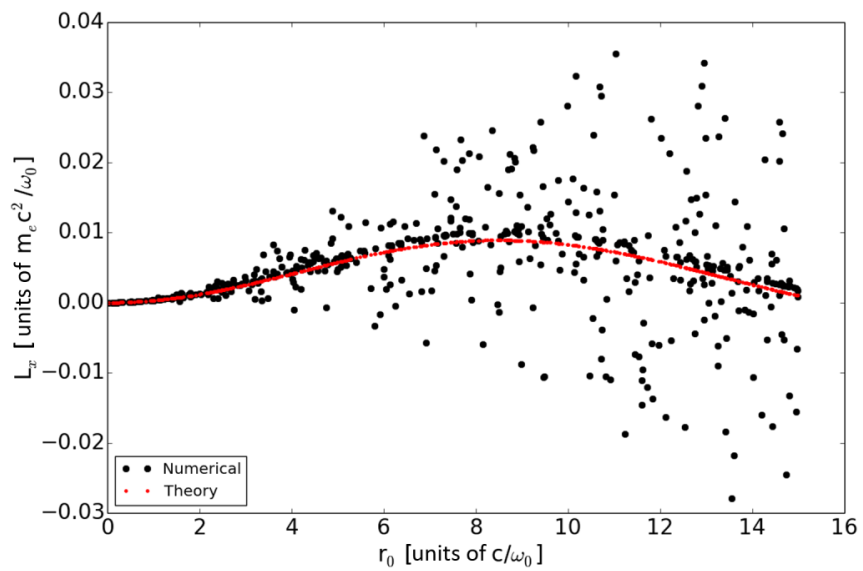

FIG. 14. $L_{x}$ versus the electron initial radial position computed with the numerical integration of motion equations (black circle) and with the perturbative formula given in Eq. (14) (red curve) for $a_{0}=$ 0.1 and $l=-1, \tau=6 \tau_{0}, w_{0}=2 \mu \mathrm{m}$.

first-order time derivative is $\dot{L}_{x}^{(1)}=r_{0} \dot{p}_{\theta}^{(1)}$, and then writes:

$$
L_{x}^{(1)}=-\alpha a_{0} r_{0} l f\left(r_{0}\right) g(t) \sin \left(t-\phi_{0}\right)
$$

Integrated over one laser optical period, we obtain $\left\langle L_{x}^{(1)}\right\rangle_{\tau_{0}}=$ 0 . Thus, the first-order term of electron longitudinal OAM does not contribute to the laser to electron angular momentum transfer.

The second-order term of electron longitudinal OAM solves

$$
\begin{aligned}
\dot{L}_{x}^{(2)}= & r^{(1)} \dot{p}_{\theta}^{(1)}-r_{0} p_{x}^{(1)} B_{r}+r_{0} p_{r}^{(1)} B_{x}-r_{0} \frac{d E_{\theta}}{d \theta}\left(\theta^{(1)}-\theta_{0}\right) \\
& -r_{0} \frac{d E_{\theta}}{d x}\left(x^{(1)}-x_{0}\right)-r_{0} \frac{d E_{\theta}}{d r}\left(r^{(1)}-r_{0}\right) .
\end{aligned}
$$

Integrated over pulse duration, the first four terms in the righthand side of Eq. (B9) are zero, so we only keep the last two

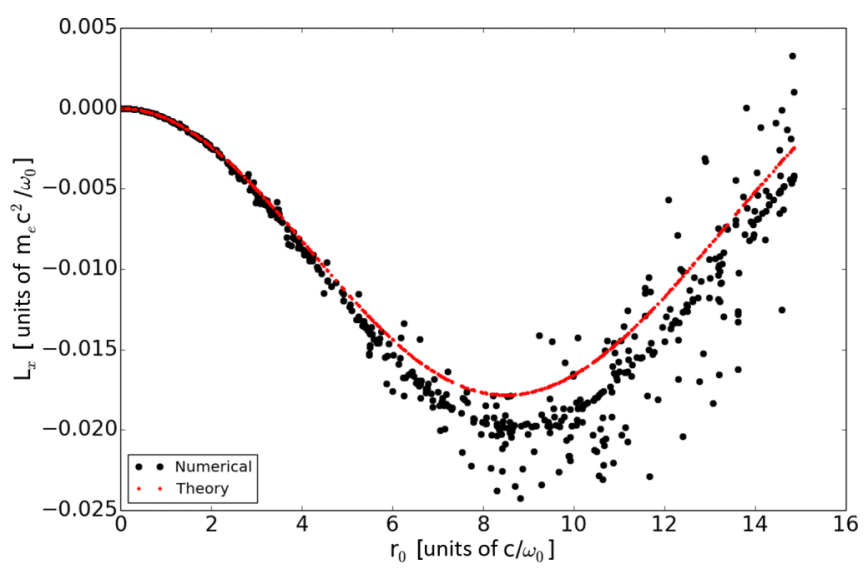

FIG. 15. $L_{x}$ versus the electron initial radial position computed with the numerical integration of motion equations (black circle) and with the perturbative formula given in Eq. (14) (red curve) for $a_{0}=$ 0.1 and $l=1, \tau=12 \tau_{0}, w_{0}=2 \mu \mathrm{m}$. 
terms

$$
\begin{aligned}
\dot{L}_{x}^{(2)}=- & r_{0} \frac{d E_{\theta}}{d r}\left(r^{(1)}-r_{0}\right)-r_{0} \frac{d E_{\theta}}{d x}\left(x^{(1)}-x_{0}\right), \\
\dot{L}_{x}^{(2)}= & -\left.\alpha l a_{0}^{2} \frac{1}{2} r_{0} \frac{d f^{2}(r)}{d r}\right|_{r=r_{0}} g^{2}(t) \cos ^{2}\left(t-\phi_{0}\right) \\
& -\alpha l a_{0}^{2} f^{2}\left(r_{0}\right) g^{2}(t) \sin ^{2}\left(t-\phi_{0}\right) .
\end{aligned}
$$

By integrating Eq. (B11) over the laser pulse duration, we obtain the analytical expression of $L_{x}^{(2)}$ given in Eq. (14). It predicts that the electron angular momentum is linearly dependent on the pulse duration and on the laser OAM.

Figure 13 draws the electron $L_{x}$ distribution versus their initial radial position computed with the numerical integration of motion equations (black circle) and with the perturbative expression given in Eq. (14) (red cuve) for $a_{0}=0.1, l=$ $1, \tau=6 \tau_{0}, w_{0}=2 \mu \mathrm{m}$. We observe a perfect agreement between both curves. As predicted by Eq. (14), the $L_{x}$ values are opposite to the laser OAM value, and are maximal in amplitude near $w_{0} / \sqrt{2}$. This confirms that the electron angular momentum originates from their longitudinal and radial motion in the laser field.

In Fig. 14, the laser OAM is now set to $l=-1$. We observe that the electrons gain positive angular momentum, but with absolute values equal to the previous case.

Equation (14) predicts a linear dependence with the pulse duration. This is confirmed in Fig. 15 where the pulse duration is two times larger.
[1] E. G. Zweibel and C. Heiles, Magnetic fields in galaxies and beyond, Nature 385, 131 (1997).

[2] T. Toncian, M. Borghesi, J. Fuchs, E. d'Humières, P. Antici, P. Audebert, E. Brambrink, C. A. Cecchetti, A. Pipahl, L. Romagnani, O. Willi, and R. Hertel, Ultrafast laser-driven microlens to focus and energy-select Mega-Electron Volt protons, Science 312, 410 (2006).

[3] V. I. Berezhiani, S. M. Mahajan, and N. L. Shatashvili, Theory of magnetic field generation by relativistically strong laser radiation, Phys. Rev. E 55, 995 (1997).

[4] M. G. Haines, Generation of an Axial Magnetic Field from Photon Spin, Phys. Rev. Lett. 87, 135005 (2001).

[5] R. Hertel, Theory of the inverse Faraday effect in metals, J. Magn. Magn. Mater. 303, L1 (2006).

[6] G. Shvets, N. J. Fisch, and J.-M. Rax, Magnetic field generation through angular momentum exchange between circularly polarized radiation and charged particles, Phys. Rev. E 65, 046403 (2002).

[7] N. Naseri, V. Yu. Bychenkov, and W. Rozmus, Axial magnetic field generation by intense circularly polarized laser pulses in underdense plasmas, Phys. Plasmas 17, 083109 (2010).

[8] T. V. Liseykina, S. V. Popruzhenko, and A. Macchi, Inverse Faraday effect driven by radiation friction, New J. Phys. 18, 072001 (2016).

[9] Z. Lécz, A. Andreev, and A. Seryi, Plasma rotation with circularly polarized laser pulse, Laser Part. Beams 34, 31 (2015).

[10] Ph. Korneev, E. d'Humières, and V. Tikhonchuk, Gigagaussscale quasistatic magnetic field generation in a snail-shaped target, Phys. Rev. E 91, 043107 (2015).

[11] Ph. Korneev, V. Tikhonchuk, and E. d'Humières, Magnetization of laser-produced plasma in a chiral hollow target, New J. Phys. 19, 033023 (2017).

[12] S. Ali, J. R. Davies, and J. T. Mendonca, Inverse Faraday Effect with Linearly Polarized Laser Pulses, Phys. Rev. Lett. 105, 035001 (2010).
[13] Z. Lécz, I. V. Konoplev, A. Seryi, and A. Andreev, Gigagauss solenoidal magnetic field inside bubbles excited in under-dense plasma, Nature: Scientific Reports 6, 36139 (2016).

[14] Z. Najmudin, M. Tatarakis, A. Pukhov, E. L. Clark, R. J. Clarke, A. E. Dangor, J. Faure, V. Malka, D. Neely, M. I. K. Santala, and K. Krushelnick, Measurements of the Inverse Faraday Effect from Relativistic Laser Interactions with an Underdense Plasma, Phys. Rev. Lett. 87, 215004 (2001).

[15] J. Deschamps, M. Fitaire, and M. Lagoutte, Inverse Faraday Effect in a Plasma, Phys. Rev. Lett. 25, 1330 (1970).

[16] Y. Horovitz, S. Eliezer, A. Ludmirsky, Z. Henis, E. Moshe, R. Shpitalnik, and B. Arad, Measurements of Inverse Faraday Effect and Absorption of Circularly Polarized Laser Light in Plasmas, Phys. Rev. Lett. 78, 1707 (1997).

[17] L. Allen, M. W. Beijersbergen, R. J. C. Spreeuw, and J. P. Woerdman, Orbital angular momentum of light and the transformation of Laguerre-Gaussian laser modes, Phys. Rev. A 45, 8185 (1992).

[18] W. Wang, B. Shen, X. Zhang, L. Zhang, Y. Shi, and Z. Xu, Hollow screw-like drill in plasma using an intense LaguerreGaussian laser, Sci. Rep. 5, 8274 (2015).

[19] R. Li, X. Feng, D. Zhang, K. Cui, F. Liu, and Y. Huang, Radially polarized orbital angular momentum beam emitter based on shallow-ridge silicon microring cavity, IEEE Photonics J. 6, 2200710 (2014).

[20] I. Thiele, S. Skupin, and R. Nuter, Boundary conditions for arbitrarily shaped and tightly focused laser pulses in electromagnetic codes, J. Comput. Phys. 321, 1110 (2016).

[21] A. E. Siegman, LASERS (University Science Books, Sausalito, CA, 1986).

[22] R. Nuter and V. T. Tikhonchuk, Prepulse suppression and optimization of backward Raman amplification with a chirped pump laser beam, Phys. Rev. E 87, 043109 (2013).

[23] J. D. Jackson, Classical Electrodynamics (Wiley, New York, 1998). 